Acta Cryst. (1992). C48, 393-395

\title{
Structure of 2-[(4-Cyanophenyl)methylene]-1,3-dithiole-4,5-dicarboxylic Acid, Dimethyl Ester
}

\author{
By Frank C. J. M. van VegGeL* \\ Akzo Research Laboratories Arnhem, Department of Organic and Polymer Chemistry, PO Box 9300, \\ 6800 SB Arnhem, The Netherlands \\ AND Sybolt HaRkema \\ Laboratory of Chemical Physics, University of Twente, PO Box 217, 7500 AE Enschede, The Netherlands
}

(Received 12 June 1991; accepted 23 July 1991)

Abstract. $\mathrm{C}_{15} \mathrm{H}_{11} \mathrm{NO}_{4} \mathrm{~S}_{2}, M_{r}=333.39$, triclinic, $P \overline{1}, a$ $=20.611$ (7) $, \quad b=9.452(5), \quad c=7.856(4) \AA, \quad \alpha=$ $98.52(3), \quad \beta=96.05(2), \quad \gamma=93.0(3)^{\circ}, \quad V=$ $1502(2) \AA^{3}, Z=4, D_{x}=1.475 \mathrm{~g} \mathrm{~cm}^{-3}, \lambda($ Mo $K \alpha)=$ $0.7107 \AA, \mu=3.55 \mathrm{~cm}^{-1}, F(000)=688, T=293 \mathrm{~K}$, $R=0.051 \quad(w R=0.059)$ for 3388 reflections. The asymmetric unit contains two independent molecules which have a similar conformation. The dithiole ring is planar in both molecules and makes an angle with the phenyl ring of 2.1 and $0.6^{\circ}$, respectively. Thus the methylene-1,3-dithiole unit (electron donor) has a maximum $\pi$ overlap with the phenyl ring, that bears a $p$-cyano group (electron acceptor). In both molecules the methyl esters are planar: one is almost parallel to the dithiole ring (angles of 9.8 and $8.5^{\circ}$, respectively) and the other makes angles of 54.2 and $63.8^{\circ}$, respectively.

Experimental. The title molecule (see scheme) was prepared following the procedure reported by Mulvaney \& Chang (1980) for 2-(phenylmethylene)-1,3dithiole-4,5-dicarboxylic acid, dimethyl ester, starting from $p$-cyanobenzaldehyde, dimethyl acetylenedicarboxylate and carbon disulfide in the presence of $(n-\mathrm{Bu})_{3} \mathrm{P}$ in diethyl ether at $250 \mathrm{~K}$. Orange-red cubes were obtained by recrystallization from acetonitrile, dimensions $0.4 \times 0.5 \times 0.5 \mathrm{~mm}$.

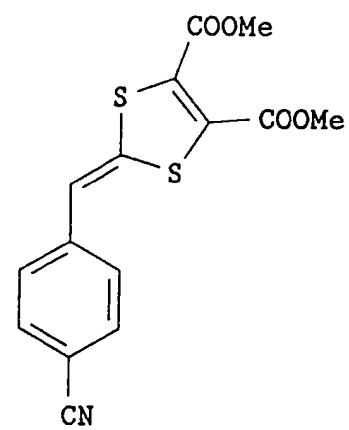

* To whom correspondence should be addressed.

0108-2701/92/020393-03\$03.00
Table 1. Positional parameters and their estimated standard deviations

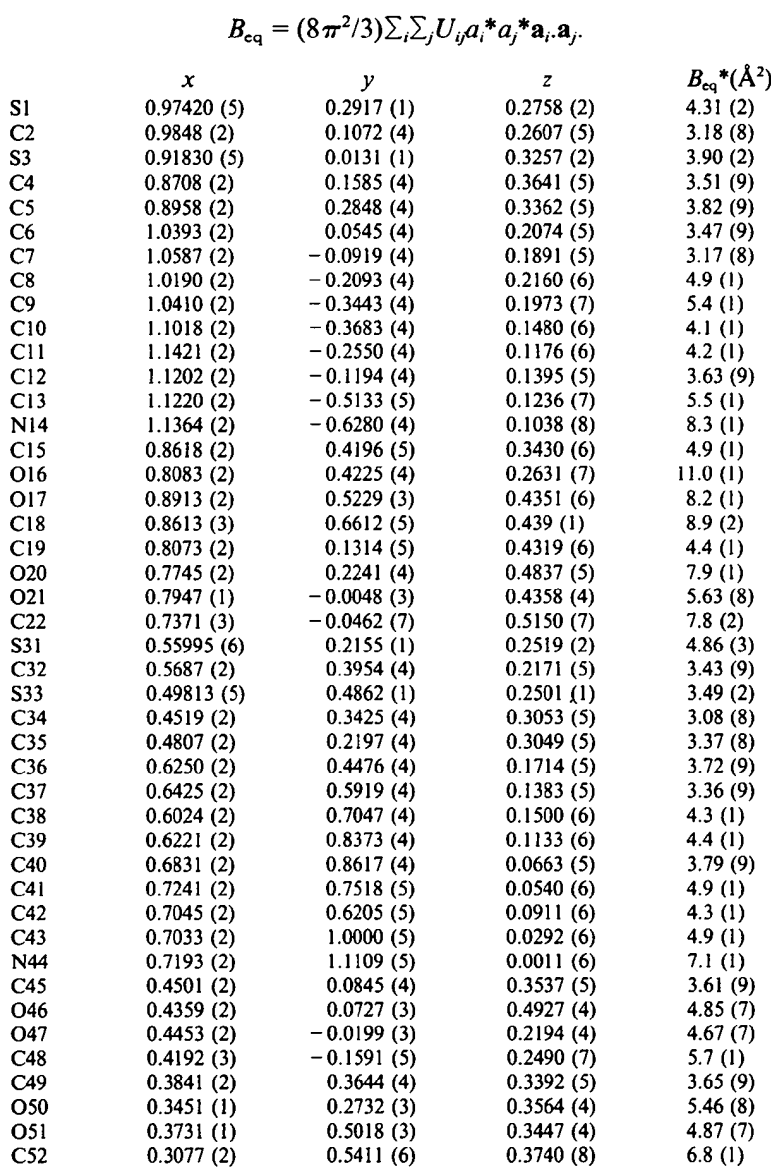

The cell parameters were obtained by least-squares fit using 25 centered reflections with $6<\theta<25^{\circ}$. An Enraf-Nonius CAD-4 diffractometer was used for data collection, 5292 unique reflections, $-24 \leq h \leq$ $24, \quad-11 \leq k \leq 11, \quad 0 \leq l \leq 9, \quad[(\sin \theta) / \lambda]_{\max }=$ $0.5947 \AA^{-1}$. Intensities were measured in the $\omega / 2 \theta$

(C) 1992 International Union of Crystallography 
Table 2. Bond distances $(\AA)$ and angles $\left(^{\circ}\right)$

Numbers in parentheses are estimated standard deviations in the least significant digits.

\begin{tabular}{|c|c|c|c|}
\hline $\mathrm{S} 1-\mathrm{C} 2$ & $1.757(4)$ & $\mathrm{S} 31-\mathrm{C} 32$ & $1.765(4)$ \\
\hline $\mathrm{S} 1-\mathrm{C} 5$ & $1.733(4)$ & $\mathrm{S} 31-\mathrm{C} 35$ & $1.730(4)$ \\
\hline $\mathrm{C} 2-\mathrm{S} 3$ & $1.757(5)$ & C32-S33 & $1.750(5)$ \\
\hline $\mathrm{C} 2-\mathrm{C} 6$ & $1.334(6)$ & $\mathrm{C} 32-\mathrm{C} 36$ & $1.342(6)$ \\
\hline S3-C4 & $1.737(4)$ & $\mathrm{S} 33-\mathrm{C} 34$ & $1.752(4)$ \\
\hline $\mathrm{C} 4-\mathrm{C} 5$ & $1.332(6)$ & C $34-\mathrm{C} 35$ & $1.330(5)$ \\
\hline $\mathrm{C} 4-\mathrm{C} 19$ & $1.490(7)$ & C $34-C 49$ & $1.469(6)$ \\
\hline $\mathrm{CS}-\mathrm{C} 15$ & $1.482(7)$ & $\mathrm{C} 35-\mathrm{C} 45$ & $1.512(6)$ \\
\hline $\mathrm{C} 6-\mathrm{C} 7$ & $1.452(5)$ & $\mathrm{C} 36-\mathrm{C} 37$ & $1.460(6)$ \\
\hline $\mathrm{C} 7-\mathrm{C} 8$ & $1.399(6)$ & C37-C38 & $1.381(6)$ \\
\hline $\mathrm{C} 7-\mathrm{C} 12$ & $1.390(6)$ & $\mathrm{C} 37-\mathrm{C} 42$ & $1.392(6)$ \\
\hline $\mathrm{C} 8-\mathrm{C} 9$ & $1.369(6)$ & $\mathrm{C} 38-\mathrm{C} 39$ & $1.377(6)$ \\
\hline $\mathrm{Cg}-\mathrm{ClO}$ & $1.371(6)$ & C39-C40 & $1.365(6)$ \\
\hline $\mathrm{ClO}-\mathrm{C} 11$ & $1.385(6)$ & $\mathrm{C} 40-\mathrm{C} 41$ & $1.373(6)$ \\
\hline $\mathrm{Cl} 0-\mathrm{Cl} 3$ & $1.445(7)$ & $\mathrm{C} 40-\mathrm{C} 43$ & $1.431(6)$ \\
\hline $\mathrm{Cl}-\mathrm{Cl} 2$ & $1.375(6)$ & $\mathrm{C} 41-\mathrm{C} 42$ & $1.366(7)$ \\
\hline $\mathrm{Cl3}-\mathrm{N} 14$ & $1.132(6)$ & $\mathrm{C} 43-\mathrm{N} 44$ & $1.142(7)$ \\
\hline $\mathrm{C} 15-016$ & $1.214(6)$ & $\mathrm{C} 45-\mathrm{O} 46$ & $1.180(5)$ \\
\hline $\mathrm{Cl} 5-017$ & $1.218(5)$ & $\mathrm{C} 45-047$ & $1.326(4)$ \\
\hline $\mathrm{O} 17-\mathrm{C} 18$ & $1.473(6)$ & $\mathrm{O} 47-\mathrm{C} 48$ & $1.456(5)$ \\
\hline $\mathrm{Cl} 9-\mathrm{O} 20$ & $1.186(6)$ & $\mathrm{C} 49-\mathrm{O} 50$ & $1.181(6)$ \\
\hline $\mathrm{C} 19-\mathrm{O} 21$ & $1.305(6)$ & $\mathrm{C} 49-\mathrm{O} 51$ & $1.326(5)$ \\
\hline $\mathrm{O} 21-\mathrm{C} 22$ & $1.458(7)$ & $\mathrm{O} 51-\mathrm{C} 52$ & $1.449(6)$ \\
\hline $\mathrm{C} 2-\mathrm{S} 1-\mathrm{C} 5$ & $96.7(2)$ & $\mathrm{C} 32-\mathrm{S} 31-\mathrm{C} 35$ & $96.6(2)$ \\
\hline $\mathrm{S} 1-\mathrm{C} 2-\mathrm{S} 3$ & $112.1(2)$ & $\$ 31-\mathrm{C} 32-\mathrm{S} 33$ & $112.3(2)$ \\
\hline $\mathrm{S} 1-\mathrm{C} 2-\mathrm{C} 6$ & 120.2 (4) & $\mathrm{S} 31-\mathrm{C} 32-\mathrm{C} 36$ & $120.1(4)$ \\
\hline $\mathrm{S} 3-\mathrm{C} 2-\mathrm{C} 6$ & $127.6(3)$ & $\mathrm{S} 33-\mathrm{C} 32-\mathrm{C} 36$ & $127.6(4)$ \\
\hline $\mathrm{C} 2-\mathrm{S} 3-\mathrm{C} 4$ & $96.8(2)$ & $\mathrm{C} 32-\mathrm{S} 33-\mathrm{C} 34$ & $96.7(2)$ \\
\hline $\mathrm{S} 3-\mathrm{C} 4-\mathrm{C} 5$ & $117.0(3)$ & $\mathrm{S} 33-\mathrm{C} 34-\mathrm{C} 35$ & $116.6(3)$ \\
\hline $\mathrm{S} 3-\mathrm{C} 4-\mathrm{C} 19$ & $116.9(3)$ & $\mathrm{S} 33-\mathrm{C} 34-\mathrm{C} 49$ & $117.9(3)$ \\
\hline $\mathrm{C} 5-\mathrm{C} 4-\mathrm{C} 19$ & $126.1(4)$ & $\mathrm{C} 35-\mathrm{C} 34-\mathrm{C} 49$ & $125.4(4)$ \\
\hline $\mathrm{S} 1-\mathrm{Cs}-\mathrm{C} 4$ & $117.3(3)$ & $\mathrm{S} 31-\mathrm{C} 35-\mathrm{C} 34$ & $117.7(4)$ \\
\hline $\mathrm{S} 1-\mathrm{C} 5-\mathrm{C} 15$ & $116.2(4)$ & S $31-C 35-C 45$ & $117.3(3)$ \\
\hline $\mathrm{C} 4-\mathrm{C} 5-\mathrm{C} 15$ & $126.4(4)$ & $\mathrm{C} 34-\mathrm{C} 35-\mathrm{C} 45$ & 124.9 (4) \\
\hline $\mathrm{C} 2-\mathrm{C} 6-\mathrm{C} 7$ & $129.5(4)$ & $\mathrm{C} 32-\mathrm{C} 36-\mathrm{C} 37$ & $129.0(4)$ \\
\hline C6 $-\mathrm{C} 7-\mathrm{C} 8$ & $124.1(4)$ & $\mathrm{C} 36-\mathrm{C} 37-\mathrm{C} 38$ & $125.3(4)$ \\
\hline $\mathrm{C} 6-\mathrm{C} 7-\mathrm{Cl2}$ & $118.8(3)$ & $\mathrm{C} 36-\mathrm{C} 37-\mathrm{C} 42$ & 118.4 (4) \\
\hline $\mathrm{C} 8-\mathrm{C} 7-\mathrm{C} 12$ & $117.1(4)$ & $\mathrm{C} 38-\mathrm{C} 37-\mathrm{C} 42$ & $116.3(4)$ \\
\hline $\mathrm{C} 7-\mathrm{C} 8-\mathrm{C}$ & $120.7(4)$ & $\mathrm{C} 37-\mathrm{C} 38-\mathrm{C} 39$ & $121.9(4)$ \\
\hline $\mathrm{C} 8-\mathrm{C} 9-\mathrm{C} 10$ & $120.9(5)$ & $\mathrm{C} 38-\mathrm{C} 39-\mathrm{C} 40$ & $120.3(4)$ \\
\hline $\mathrm{C} 9-\mathrm{Cl}-\mathrm{Cl1}$ & $119.9(4)$ & $\mathrm{C} 39-\mathrm{C} 40-\mathrm{C} 41$ & $119.0(4)$ \\
\hline $\mathrm{C}-\mathrm{C} 10-\mathrm{C} 13$ & $119.0(4)$ & $\mathrm{C} 39-\mathrm{C} 40-\mathrm{C} 43$ & $120.0(4)$ \\
\hline $\mathrm{C} 11-\mathrm{Cl}-\mathrm{C} 13$ & $121.1(4)$ & $\mathrm{C} 41-\mathrm{C} 40-\mathrm{C} 43$ & $120.9(5)$ \\
\hline $\mathrm{ClO}-\mathrm{Cl1}-\mathrm{Cl2}$ & $118.9(4)$ & $\mathrm{C} 40-\mathrm{C} 41-\mathrm{C} 42$ & $120.4(5)$ \\
\hline $\mathrm{C} 7-\mathrm{C} 12-\mathrm{Cl1}$ & $122.4(4)$ & $\mathrm{C} 37-\mathrm{C} 42-\mathrm{C} 41$ & $121.9(4)$ \\
\hline $\mathrm{Cl}-\mathrm{Cl} 3-\mathrm{N} 14$ & $178.5(5)$ & $\mathrm{C} 40-\mathrm{C} 43-\mathrm{N} 44$ & $179.5(6)$ \\
\hline $\mathrm{C} 5-\mathrm{C} 15-016$ & $121.0(4)$ & $\mathrm{C} 35-\mathrm{C} 45-\mathrm{O} 46$ & 124.9 (3) \\
\hline $\mathrm{C} 5-\mathrm{C} 15-\mathrm{O} 17$ & $114.8(4)$ & $\mathrm{C} 35-\mathrm{C} 45-\mathrm{O} 47$ & $109.5(3)$ \\
\hline $016-\mathrm{Cl5}-017$ & $124.2(5)$ & $046-\mathrm{C} 45-047$ & $125.5(4)$ \\
\hline $\mathrm{Cl5}-017-\mathrm{Cl} 18$ & $116.9(4)$ & $\mathrm{C} 45-\mathrm{O} 47-\mathrm{C} 48$ & $115.9(3)$ \\
\hline $\mathrm{C} 4-\mathrm{C} 19-\mathrm{O} 20$ & $123.4(4)$ & $\mathrm{C} 34-\mathrm{C} 49-\mathrm{O} 50$ & $125.2(4)$ \\
\hline $\mathrm{C} 4-\mathrm{C} 19-\mathrm{O} 21$ & $111.6(4)$ & $\mathrm{C} 34-\mathrm{C} 49-\mathrm{O} 51$ & $109.7(3)$ \\
\hline $\mathrm{O} 20-\mathrm{C} 19-\mathrm{O} 21$ & $125.1(4)$ & $\mathrm{O} 50-\mathrm{C} 49-\mathrm{O} 51$ & $125.1(4)$ \\
\hline $\mathrm{C} 19-\mathrm{O} 21-\mathrm{C} 22$ & $117.8(4)$ & $\mathrm{C} 49-\mathrm{O} 51-\mathrm{C} 52$ & $116.6(3)$ \\
\hline
\end{tabular}

scan mode [scan speed $4.1^{\circ} \mathrm{s}^{-1}$, scan width $(\theta) 1+$ $\left.0.34 \tan \theta^{\circ}\right]$. Decay of three control reflections, measured every hour, was $<1 \%$. Correction was made for Lorentz and polarization factors. Number of observed reflections $3388\left[F_{o}^{2}>3 \sigma\left(F_{o}^{2}\right)\right]$. No absorption correction was used.

Structure solved by direct methods (Germain, Main \& Woolfson, 1971) and refined by full-matrix least squares. Weights for each reflection in the refinement (on $F$ ) were calculated from $w=4 F_{o}^{2}$ / $\sigma^{2}\left(F_{o}^{2}\right), \sigma^{2}\left(F_{o}^{2}\right)=\sigma^{2}(I)+\left(p F_{o}^{2}\right)^{2}$; the value of the instability factor $p$ was determined as $0.04 . \mathrm{H}$ atoms were placed in calculated positions and were refined. The number of parameters refined was 486: scale factor, isotropic extinction factor $g\left[F_{\text {corr }}=F_{c} /(1+\right.$ $g I_{c}$ ); final value $\left.2.1 \times 10^{-7}\right]$, positional and aniso- tropic thermal parameters for non- $\mathrm{H}$ atoms and positional and isotropic thermal parameters for $\mathrm{H}$ atoms.

Refinement converged at $R=0.051, w R=0.059$, $S=1.88,(\Delta / \sigma)_{\max }=0.2$. Largest peak on the final difference Fourier map $0.40 \mathrm{e} \AA^{-3}$. All calculations were performed using $S D P$ (Frenz \& Associates, Inc., 1983). Atomic scattering factors from International Tables for X-ray Crystallography (1974, Vol. IV). Atomic parameters of non- $\mathrm{H}$ atoms are given in Table $1 .^{*}$ Bond distances and bond angles are given in Table 2. Atom numbering of the title compound is shown in Fig. 1.

Related literature. To the best of our knowledge no crystal structure of a para-substituted aromatic ring with a 4,5-functionalized methylene-1,3-dithiole moiety as electron donor and a cyano group as electron acceptor has been published. However, some related structures have been determined. The crystal structure of a dithiinofulvene derivative with a methylene-1,3-dithiole-4,5-dicarboxylic

* Lists of structure factors, anisotropic thermal parameters, $\mathrm{H}$-atom parameters and complete lists of bond lengths and angles have been deposited with the British Library Document Supply Centre as Supplementary Publication No. SUP 54552 (38 pp.). Copies may be obtained through The Technical Editor, International Union of Crystallography, 5 Abbey Square, Chester CH1 2HU, England. [CIF reference: AL0486]

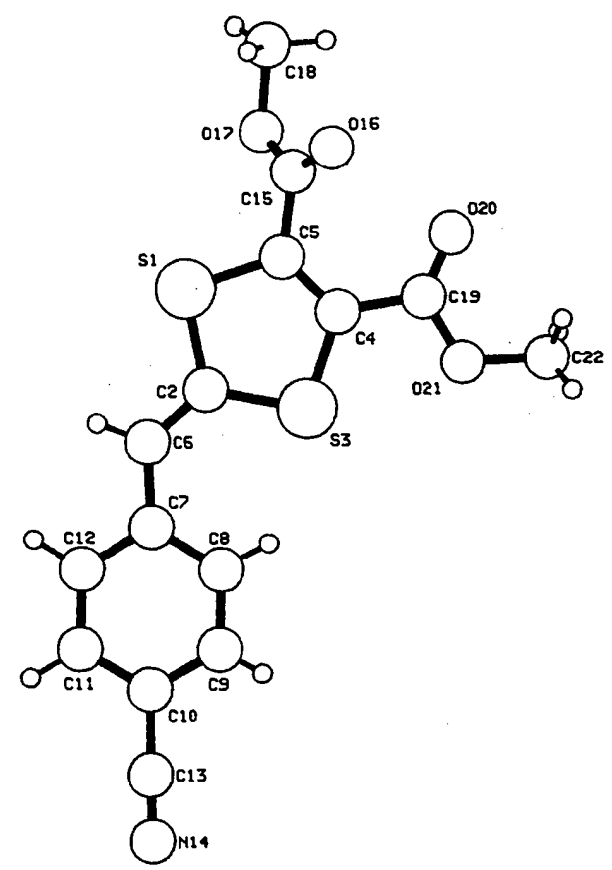

Fig. 1. View of one independent molecule with the atomnumbering scheme. The atom numbers in molecule (2) are obtained by adding 30 to those of molecule (1). 
acid, dimethyl ester unit has been reported by Lakshmikantham, Cava \& Carroll (1984). A niobium complex with a methylene-1,3-dithiole-4,5-bis(trifluoromethyl) unit has been published by Amaudrut, Sala-Pala, Guerchais \& Mercier (1990). Beer, Frew, Johnson \& Paul (1970) have reported an isothiathiophten containing a methylene-1,3-dithiole4-(4-bromophenyl) unit. The structure of (pivaloylmethylene)-1,3-dithiole-4-pivaloyl has been reported by Galloy, Declercq \& Van Meerssche (1978). A 4,5-unsubstituted methylene-1,3-dithiole derivative has been reported by Barnes, Paton \& Nicholls (1982). The structures of the related compounds mentioned here are less accurate than that reported in this paper.

\section{References}

Amaudrut, J., Sala-Pala, J., Guerchais, J. E. \& Mercier, R. (1990). J. Organomet. Chem. 391, 61-80.

Barnes, J. C., Paton, J. D. \& Nicholls, B. H. (1982). Acta Cryst. B38, 1525-1529.

Beer, R. J. S., Frew, D., Johnson, P. L. \& Paul, I. C. (1970). J. Chem. Soc. Chem. Commun. pp. 154-155.

Frenz, B. A. \& Associates, INC. (1983). SDP Structure Determination Package. College Station, Texas, USA.

Galloy, J., DeclercQ, J.-P. \& Van Meerssche, M. (1978). Acta Cryst. B34, 975-978.

Germain, G., Main, P. \& Woolfson, M. M. (1971). Acta Cryst. A27, 368-376.

Lakshmikantham, M. V., Cava, M. P. \& Carroll, P. J. (1984). J. Org. Chem. 49, 726-728.

Mulvaney, J. E. \& Chang, D. M. (1980). Macromolecules, 13, 240-243.

Acta Cryst. (1992). C48, 395-j96

\title{
Structure of Methyl 2-(2,5-Dihydro-2-oxo-3-phenyl-5-furyl)lactate
}

\author{
By Rosemary C. Hynes \\ Institute for Environmental Chemistry, National Research Council of Canada, Ottawa K1A 0R6, Canada
}

(Received 2 May 1991; accepted 9 July 1991)

\begin{abstract}
C}_{14} \mathrm{H}_{14} \mathrm{O}_{5}, M_{r}=262.26$, monoclinic, $P 2_{1} / n$, $a=11.319(3), b=6.5115(15), c=18.105(5) \AA, \beta=$ $105.660(20)^{\circ}, \quad V=1284.8(6) \AA^{3}, \quad Z=4, \quad D_{x}=$

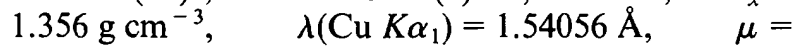
$8.26 \mathrm{~cm}^{-1}, F(000)=553.89, T=295 \mathrm{~K}, R=0.036$, $w R=0.033$ for 1692 reflections with $I>2.5 \sigma(I)$. The structure determination established the identity of the product of a $\mathrm{Co}_{2}(\mathrm{CO})_{8}$-catalyzed carbonylation reaction with an epoxy alcohol.
\end{abstract}

Experimental. A colourless crystal of the title compound (1) was grown from THF solution. Cell parameters from 20 reflections in the $2 \theta$ range 85-90 . Data were collected on a Picker diffractometer using profile analysis (Grant \& Gabe, 1978). Three standards measured every 100 reflections were used for scaling and had an overall drop in intensity of $6 \%$. Averaging of symmetry-equivalent reflections gave a merging $R$ value of $3.3 \%$. Parameters related to crystal data and intensity collection are given in Table 1. The structure was solved by direct methods and refined by cycles of least-squares calculations. $\mathrm{H}$ atoms were located in a difference map and refined isotropically. Final atomic parameters are listed in Table 2. All non-H atoms were refined anisotropically. All computing was performed with the NRCVAX system of structure-solving programs (Gabe, Le Page, Charland, Lee \& White, 1989).

0108-2701/92/020395-02\$03.00
Scattering factors from International Tables for $X$-ray Crystallography (1974, Vol. IV).*

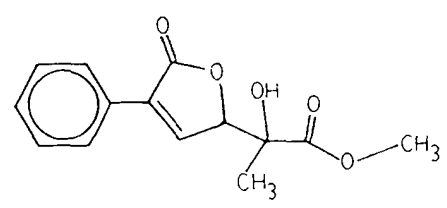

(1)

An ORTEPII plot of (1) is shown in Fig. 1. Bond lengths and angles, except those involving $\mathrm{H}$ atoms, are given in Table 3. A stereoplot showing packing of the unit cell has been included as Fig. 2. The structure consists of a planar lactone ring with maximum deviation from planarity 0.0098 (18) $\AA$, rotated slightly from the plane of the phenyl ring, with a dihedral angle of $16.73(6)^{\circ}$. The diastereomer adopts an anti configuration. The molecules are packed with the lactone rings approximately parallel to the $b$ axis. The closest intermolecular approach is $2.14(2) \AA$

* Lists of structure factors, anisotropic thermal parameters and least-squares-planes calculations have been deposited with the British Library Document Supply Centre as Supplementary Publication No. SUP 54457 (15 pp.). Copies may be obtained through The Technical Editor, International Union of Crystallography, 5 Abbey Square, Chester $\mathrm{CH} 1$ 2HU, England. [CIF reference: CR0352]

(C) 1992 International Union of Crystallography 